\title{
Research on Teaching Methods of Automatic Control Systems
}

\author{
Raofen Wang, Liping Zhang, Jianzhen Wu, Dongmei Xu, Dongbing Tong \\ School of Electronic and Electrical Engineering, Shanghai University of Engineering Science, Shanghai, China
}

Email address:

rfwangsues@163.com (Raofen Wang)

\section{To cite this article:}

Raofen Wang, Liping Zhang, Jianzhen Wu, Dongmei Xu, Dongbing Tong. Research on Teaching Methods of Automatic Control Systems. Education Journal. Vol. 5, No. 3, 2016, pp. 39-42. doi: 10.11648/j.edu.20160503.12

Received: May 9, 2016; Accepted: June 3, 2016; Published: June 17, 2016

\begin{abstract}
Automatic control systems is the specialized fundamental course of automation major. The teaching effect of this course will influence the learning of the post-requisites. It's important to improve the teaching quality of this course. This paper analyzed the problems of existing teaching methods. According to these problems, teaching reform methods were proposed to engage and motivate students' initiative, strengthen the students' capability of analyzing and solving problems.
\end{abstract}

Keywords: Automatic Control Systems, Teaching Reform, Learning Outcomes, Deep Learning, Case Study

\section{Introduction}

Automatic Control Systems (ACS) is a very important specialized fundamental course of automatic major. Also, it's the basic course of majors such as electrical information, mechanism, railway transportation, etc. With the development of science and technology, automatic control technology is extending to the application of biological, medical, environmental, economic, social life, etc. Automatic control has become an important component of the current social activities. It's important to cultivate student's ability of practice and innovation through the teaching of Automatic Control Systems.

Before learning Automatic Control Systems, students must have finished the studying of some courses, i.e. "Advanced Mathematics", "Circuit", and "Physics". The post-requisites of this course are "Modern Control Theory", "Intelligent Control", "Adaptive Control", etc. Automatic Control Systems plays an important role in control science and engineering discipline [1-3].

Automatic Control Systems is an extremely theoretical curriculum, which needs to learn and understand a number of mathematical knowledge in advance. It's hard for students to master this course. Before learning this course, students need to master the knowledge on mathematics, electricity, physics and other aspects of knowledge [4, 5]. In this course, students are required to understand the principle and mechanisms of automatic control systems through complex formulas and theorems. Also, they need the abstract thinking to handle all the knowledge.

Therefore, teachers can not only explain the theorems and formulas when teaching Automatic Control Systems, but also introduce practice cases to the students and use tools, e.g. multimedia, MATLAB, etc. to assist teaching [6]. Then, students can have intuitive understanding of theorems and formulas. Thereby, students could use automatic control theories to solve practical problems.

This paper characterized the course of Automatic Control Systems and discussed the problems existing in teaching Automatic Control Systems. Based on the authors' teaching experience and new teaching ideas and methods that are learned from Canada, the aim of this paper is to study and discuss the teaching reform methods of Automatic Control Systems.

The rest of the paper is arranged as follows. Section 2 analyzed the existing teaching methods of Automatic Control Systems. Reforms of teaching method are presented in Section 3. Section 4 gives the conclusion.

\section{Analysis of Existing Teaching Methods}

\subsection{Teaching Mode}

It's very common for a class to have about 80 students in our schools. Practice teaching methods like experiment, field 
practice, project, etc. are rarely used because of the class size. This course contains 72 lecture classes and 8 experimental classes. Lectures about theories and formulas are the common teaching methods in the university. The amount of theoretical lectures accounts for $90 \%$, which is mainly finished by way of writing on the blackboard, power point, etc. In the teaching process, there is very complicated calculation and complex derivation. Students need to complete complex calculation, proof, etc. Comparatively, the amount of experimental classes is very little. Students have little intuitive understanding of the actual automatic systems. Therefore, most of the students can't effectively link theory with practice. They don't know how to apply automatic control theories to actual systems. Students would forget what they have learned in this course after exam. In the following courses, students are still lack of understanding of the basic principles of automatic control system.

\subsection{Contents of Course}

The contents of Automatic Control Systems include basic concepts of automatic control systems, system modelling, time domain analysis, root-locus method, frequency domain analysis, correction methods of control system, etc. [7, 8] Table 1 shows the contents, topic and class arrangement of ACS.

Table 1. Contents of Automatic Control Systems.

\begin{tabular}{|c|c|c|}
\hline Contents & topic & class \\
\hline Introduction & general concepts of ACS & $1-2$ \\
\hline System modelling & SISO, block diagrams, Laplace transform, signal flow diagrams, Mason equation & $3-16$ \\
\hline Time domain analysis of linear system & performance: Test signals, indices, basic 2 nd dynamic systems, Routh method & $17-34$ \\
\hline Root locus analysis & concept and procedure, stability analysis, design and analysis with root locus & $35-50$ \\
\hline Frequency response domain & Bode and Nyquist analysis methods, stability analysis & $51-68$ \\
\hline Design of feedback control & cascade compensation, systems lead-lead/lag-lead compensation design & $69-80$ \\
\hline
\end{tabular}

The recent teaching focuses on the principles and methods. Students are required to finish complex calculations by using mathematical skills. But some parts of this course, e.g. Mason equation and root-locus method, are more suitable for computer programming or simulation than manually calculation. When we use these methods to solve problems by manual calculating, it is very difficult since a series of complex formula are needed. They are more suitable for computer assisted solution. Therefore, teachers should focus on the principle, analysis method and application for these kinds of contents.

\subsection{Teaching Method}

Considering students' demands to pass the course exam and the postgraduate exam, the current teaching methods pay more attention to explain the calculating process. It's difficult to engage or motivate students and the advantage of autonomous learning is hardly played. The teachers need to pay more attention to how to use different teaching methods to teach concept, principle, and application. Appropriate use of discussion, case study, etc. can help students to master the knowledge. Students are more likely to execute deep learning.

\section{Reform of Teaching Methods}

\subsection{Create Learning Outcomes}

Learning outcomes are statements that indicate what students will know, value or be able to do by the end of the course [9, 10]. Strategic use of learning outcomes in Automatic Control Systems can result in many potential benefits.

First, students will know what is expected of them and what they should focus on in their studying. According to a survey finished by Alan Wrihgt on "learning autonomy", the "setting goals" option was the first one that the students care about. It means that learning outcomes will promote students to take significant responsibility for their own learning. Students will be more motivated and take a deeper approach to their learning. And also, they will get better performance on assignments and tests. Because, they are better able to demonstrate things when they know exactly what they are expected to demonstrate. They spend less time trying to guess what the instructor wants and more time getting the job done.

Second, learning outcomes will help teachers to show focused and strategic teaching. Once the teacher has created learning outcomes for the course, he/she can use them to plan lessons that strategically target those outcomes. For example, if one outcome is to "calculate the response of automatic control systems to different types of inputs, like step/ramp/parabola inputs", what the teacher needs to do is to decide what need to be calculated and what methods need to be used, explain methods, facilitate, and provide feedback. On the other hand, learning outcomes can be used to create strategically-targeted and appropriate assessment methods. Teachers can assess whether students have met the learning outcomes to judge the advantage and disadvantage of teaching methods.

\subsection{Encourage Deep Learning}

Deep learning approaches connect ideas to each other, dig for meaning behind facts, ideas, and figures, and try to make sense of each part in light of the whole [11, 12]. Students who take a deep learning try to interpret information through the lenses of their own experiences and prior knowledge, and process it in a way that makes it meaningful to them. They can synthesize and use knowledge so as to analyse practice problems. There are some deep learning approaches could be used in Automatic Control Systems: 


\subsubsection{Group Discussion}

Students are required to be divided into some groups. Teachers give questions to the students. Students are required to think about the question by themselves, share their ideas and discuss with their team members. But, it's a challenge for the teachers to lead discussion. Teachers should prepare good questions, which are high level, divergent, structured and straightforward, in order to engage students. Also, the teacher must be a good facilitator who can help students learn by guiding them in productive directions, setting up conditions to inspire them to think, ask questions, and meet course learning outcomes. During good discussion, students are expected to learn from each other, facilitate the understanding of knowledge and improve their ability of teamwork. There are many other forms of discussion, like, think-pair-share, oneminute paper, etc. Teachers can try other ways of discussion to engage students to deeply learn this course.

\subsubsection{Pop Quiz}

Question is raised according to the recent explained knowledge during class. Students are expected to finish the question within a specified time. They can assess themselves and compare with each other. This method is beneficial to promote the students to focus on learning.

These methods can be introduced into this course. It will help to improve the interaction between teachers and students in the classroom. It will motivate students to learn this course deeply.

\subsection{Case Study}

Automatic Control Systems is highly theoretical for students to understand. It is necessary to select practical cases which are relevant to this course and easy to achieve to facilitate the students' understanding of knowledge [13]. For example, we can take the common RC circuit to carry out case study.

\subsubsection{Theoretical Analysis Section}

- Draw the diagram of RC circuit. Obtain the transfer function and differential equation of the circuit through theoretical analysis.

- Analyse the stability using Routh criterion.

- Calculate the steady state response and transient response.

- Take time domain analysis using frequency method.

\subsubsection{Case Verification Section}

- Draw the schematic diagram of RC circuit by using Altium Designer software.

- Carry out transfer function analysis, transient analysis, DC curve analysis, AC small signal analysis, etc.

- Modify the RC parameters of this circuit and repeat the previous step.

- Analyse the relationship between RC parameters and the results of analysis.

- Summarize the study.

This example can be extended to other kinds of case study. For example, teachers can change RC circuit to simple mechanical systems, physics systems, etc. or change the simulation platform. These kinds of case study can help students to understand theoretical derivation, synthesize key knowledge points, link knowledge with practise, apply knowledge in practice and solve actual problems.

\subsection{Improve Assessment Strategies}

Assessments are one of the key components of a constructively-aligned course. The learning outcomes specify what students should achieve, the teaching methods help them achieve those outcomes, and the assessment tasks determine whether and how well the outcomes have been achieved. Teachers can get feedbacks from students through appropriate assessment tasks. The teacher's "teaching" and the students" "learning" will form a "feedback control system". In this way, "deviation" from the teaching goal and actual effect of students' learning will be obtained. Then teachers can adjust teaching method in time. In addition, the learning interest of the students will be motivated. Students will be more active and initiative.

But in this course, the traditional assessment method is examination. Students have to finish large amount of calculation in the two hour examination in the end of the course. It's difficult to test the students' understanding of related concepts and knowledge. Also it's hard to systematically evaluate how well students master the methods of analysing and designing actual ACS. Therefore, it's necessary to improve the assessment strategies. In addition to examination, we can also use the classroom interaction, homework, practical operation and other appropriate ways to assess the learning effect in time.

\subsubsection{Feedbacks Through Interaction of Teacher and Students}

Through the exchange of teacher and students, teacher can evaluate the students' understanding about basic concepts and principles. For example, the teacher can ask students to summarize the key and difficult points at the end of each class. Or the students could be required to describe the specific concepts. Besides, online communication tools, like QQ group and blog, could be used to share learning experience and materials, answer questions, etc.

\subsubsection{Feedbacks from Practice}

In the process of case study, students can cultivate the ability to think independently and deeply. The students need to draft project plan and methods, achieve the project's requirements, summarize and report their project. The cases proposed in section 3.3 and other practical cases can be designed and studied.

Teachers can monitor the experimental process of students, i.e. check the student's plans and methods, help students to solve problems encountered in the experiment, encourage students to improve project performance, etc. In such process, teachers can evaluate the students' mastery of knowledge, the capabilities of analysing and designing practical automatic control system. 


\section{Conclusion}

This paper discussed the necessity of teaching reform of Automatic Control Systems. The problems of existing teaching methods were indicated. For the purpose of improving the teaching quality of Automatic Control Systems, four aspects of teaching reform methods, i.e. create learning outcomes, encourage deep learning, case study, and improve assessment strategies were proposed. The adoption of these methods can help students to learn this course deeply and create a positive class atmosphere. Also, there are many other teaching methods that could be used in this course. All the teachers should do is to learn education theories, research and explore teaching methods and adopt new teaching methods to their class in order to improve the teaching quality.

\section{Acknowledgment}

This work is partially supported by the Education Reform Funds of Shanghai University of Engineering Science No. p201602001 and No. K201502001.

\section{References}

[1] R. Zhang, F. Wang, and Z. Chen. "Research and practice for three-dimensional teaching system of automatic control principle course". Experimental Technology and Management. 2013, 30 (9), pp. 181-184.

[2] Y. Zhang. "Details teaching of automatic control systems course". Journal of Electrical \& Electronic Education. 2014, $36(3)$, pp. 68-70.

[3] S. Tian, G. Wu. "The Research and practice about automatic control systems". Journal of Electrical \& Electronic Education. 2008, 30 (1), pp. 112-114.
[4] S. Gu, Y. Jiang. "Exploration on bilingual-teaching of Automatic Control Theory". Advanced Materials Research. 2011, pp. 1346-1349.

[5] X. Song, Z. Song, C. liu. "Teaching reform of "automatic control theory" course for the independent college students". 2010 International Conference on E-Health Networking, Digital Ecosystems and Technologies. Hangzhou, 2010, pp. 19-22.

[6] D. Buchczik, W. Ilewicz. "Teaching of measurement procedures for speciality of measurement systems in the institute of automatic control". IMEKO TC-1 Symposium on Education in Measurement and Instrumentation 2002. 2002

[7] S. N. Norman. Control Systems Engineering, 6th ed. John Wiley and Sons Ltd., 2011.

[8] F. Golnaraghi, B. C. Kuo. Automatic Control Systems, 9th ed., Wiley, 2002.

[9] M. K. Potter, E. Kustra. "A primer on learning outcomes and the SOLO taxonomy". Windsor, ON: University of Windsor. 2012.

[10] X. Wang, Y. Su, S. Cheung, et al. "Does outcomes based teaching and learning make a difference in students' learning approach?"

[11] M. K. Potter, E. Kustra. "A primer on deep and surface learning". Windsor, ON: University of Windsor. 2012.

[12] B. Offir, Y. Lev, R. Bezalel. "Surface and deep learning processes in distance education: Synchronous versus asynchronous systems". Computer \& Education. 2008, 51 (3), pp. 1172-1183.

[13] K. Sakagami, F. Satoh, A. Omoto. "A case study of introductory teaching method for architectural/ environmental acoustics using a smartphone". Acoustical Science and Technology. 2013, 34 (3), pp. 209-211. 